\title{
Calbindin-D9k as a sensitive molecular biomarker for evaluating the synergistic impact of estrogenic chemicals on GH3 rat pituitary cells
}

\author{
THUY T.B. VO ${ }^{1,2^{*}}$, BEUM-SOO AN $^{1 *}$, HYUN YANG $^{1}$, EUI-MAN JUNG ${ }^{1}$, INHO HWANG $^{1}$ and EUI-BAE JEUNG ${ }^{1}$ \\ ${ }^{1}$ Laboratory of Veterinary Biochemistry and Molecular Biology, College of Veterinary Medicine, \\ Chungbuk National University, Cheongju, Chungbuk, Republic of Korea; ${ }^{2}$ Institute of Biotechnology, \\ Vietnam Academy of Science and Technology, Hanoi, Vietnam
}

Received June 12, 2012; Accepted August 7, 2012

DOI: $10.3892 /$ ijmm.2012.1122

\begin{abstract}
Various endocrine-disrupting chemicals (EDCs) such as bisphenol A (BPA), alkylphenols [4-nonylphenol (NP) and 4-tert octylphenol (OP)] and isobutylparaben (IBP) are a constant concern due to their widespread distribution. It has been reported that some combinations of hormonedisrupting chemicals are much more powerful than any of the chemicals alone. In this study, we measured the expression of an estrogenic biomarker gene, calbindin-D9k (CaBP-9k), and progesterone receptor (PR) to evaluate the individual or combined estrogenic activity of BPA, NP, OP and IBP in GH3 rat pituitary cells. Most doses of the individual compounds and all the doses of the combined chemicals significantly increased CaBP-9k and PR mRNA and protein expression compared to the vehicle (except for PR expression after treatment with OP and NP at $\left.10^{-7} \mathrm{M}\right)$. Of note, high doses $\left(10^{-6}\right.$ and $\left.10^{-5} \mathrm{M}\right)$ of the EDC combinations increased the translational and transcriptional levels of $C a B P-9 k$ by 1.3 - to 2.4 -fold compared to each individual equivalent concentrations of EDCs. To determine whether the increased $C a B P-9 k$ gene expression was induced via intracellular estrogen receptor (ER), we blocked ER signaling using fulvestrant, an ER antagonist. The results showed that fulvestrant significantly reversed the $C a B P-9 k$ and PR upregulation following treatment with individual EDCs or their combinations. Taken together, we conclude that combinations of BPA, NP, OP and IBP in GH3 rat pituitary cells have synergistic estrogenic activities mediated by ER signaling. In addition, the expression of the $C a B P-9 k$ gene may be used as a biomarker to assess the synergistic effects of EDCs in vitro.
\end{abstract}

Correspondence to: Dr Eui-Bae Jeung, Laboratory of Veterinary Biochemistry and Molecular Biology, College of Veterinary Medicine, Chungbuk National University, 12 Gaesin-dong, Heungduk-gu, Cheongju, Chungbuk 361-763, Republic of Korea

E-mail: ebjeung@chungbuk.ac.kr

*Contributed equally

Key words: calbindin-D9k, bisphenol A, 4-tert-octylphenol, 4-nonylphenol, isobutylparaben, synergistic effects, GH3 cells

\section{Introduction}

Evaluating the toxicity of combinations endocrine-disrupting chemicals (EDCs) is one of the most important toxicological issues. A previous study on the multiplicity of xenobiotics found in the environment have demonstrated the need to develop methods for evaluating the risk associated with different chemical combinations (1). The synergistic effects of EDCs have been previously reported (2); however, the mechanisms responsible for these interactions remain elusive. An assessment of the hazards associated with each of these chemicals alone has indicated that they have negligible risks (3). Nevertheless, the evaluation of each chemical individually does not clarify its effect when used in combination. Understanding the combined effects of EDCs may lead to an estimation of the hazards that exist under physiological conditions (4).

Many environmental chemicals classified as EDCs have been found to potentially disturb the endocrine system and organs that respond to endocrine signals (5). Among these EDCs, bisphenol A (BPA), 4-nonylphenol (NP), 4-tert octylphenol (OP) and isobutylparaben (IBP) are being produced and utilized at high levels, and are also found in high levels in the environment. Furthermore, only very limited data are available on the additive or synergistic estrogenic effects of these compounds. BPA is widely used for the manufacture of polycarbonate plastics and resins that are used as linings for food and beverage containers, and as dental sealants. Alkylphenolic compounds, including NP and OP, are components of soaps, paints, herbicides and pesticides, and are also used as additives in plastics (6). Parabens are widely utilized as preservatives in food, cosmetics and pharmaceutical products (7).

Although the estrogenic activity of EDCs alone is weak, the combined exposure to EDCs or industrial chemicals can induce additional burden to the body (8). Some of these compounds may have the ability to generate genomic instability rather than estrogenicity via non-classical pathways of the estrogen receptor (ER). For example, BPA, NP and OP alter cell cycle kinetics, induce DNA damage and produce telomeric associations (9). Previously, Roy et al (8) indicated that alkylphenols, including BPA are capable of producing mutations in the mitochondrial or nuclear genome by inhibiting DNA replication. When fish are 
exposed to alkylphenolic compounds, the expression of $R$-ras genes ( $R$-ras $1, R$-ras 2 and $R$-ras 3$)$ is induced (10). In addition, the upregulation of $N$-ras in the gonads, intestine and liver has been observed in hermaphrodite fish (K. marmoratus) following exposure to BP, NP and OP (11). Other studies have found that BPA and NP affect the central nervous system during embryonic development $(12,13)$. Moreover, exposure to BPA and NP during the developmental stage results in a marked influence on neuronal vulnerability and synaptogenesis through mechanisms other than the ER-mediated pathway (14). Similar to many other xenoestrogens, parabens can mimic the effects of endogenous estrogen. Parabens have been shown to bind to ERs in the rodent uterus and induce estrogen-regulated gene expression in yeast cells (15). Furthermore, an association between the use of cosmetics containing parabens and an increased incidence of breast cancer in humans and animals has been reported $(16,17)$.

The additive or synergistic burden of estrogenicity and genomic instability can produce more detrimental effects compared to estrogenic action alone. The estrogenic effect of the combination of $17 \beta$-estradiol (E2) and BPA has been shown to result in higher vitellogenin contents than treatment with a single treatment at identical concentrations in a time- and dose-dependent manner in male Chinese loaches (18). The synergistic effect of triiodothyronine plus BPA on growth hormone release is due to post-translational regulation, and BPA can disrupt thyroid hormone function in GH3 cells (19). In our previous studies, the synergistic effects of BPA and OP (20) or paraben (21) were evaluated. The results demonstrated that the combined exposure to these chemicals increases their synergistic estrogenic activities in vitro. In addition, the presence of combinations of industrial chemicals and parabens (e.g., Methyparaben and propylparaben in combination with phthalates) was observed in the majority of body care cosmetics (22). However, there are very few reports on the additive or synergistic effects of BPA, NP, OP and IBP in vitro or in vivo.

The gene that we used to measure the effects of EDCs in the present study, encoding the cytosolic calcium-binding protein, calbindin-D9k (CaBP-9k), has been extensively utilized for detecting estrogenic compounds in vitro and in vivo (23). CaBP-9k expression is rapidly and highly induced by estrogenic compounds (e.g., parabens, BPA, OP, NP, phthalates, methoxychlor, diethylstilbestrol and genistein) both in vivo and in vitro, possibly through an ER-mediated pathway (24-29). In the present study, we developed an assay for evaluating the synergistic impact of the exposure to combinations of EDCs on $\mathrm{GH} 3$ rat pituitary cells using the $C a B P-9 k$ gene as an estrogenic biomarker.

\section{Materials and methods}

Reagents and chemicals. E2, BPA and NP were obtained from Sigma-Aldrich (St. Louis, MO). OP was purchased from Fluka Chemie (Seoul, Korea). IBP was obtained from Tokyo Kasei Kogyo Co., Ltd. (Tokyo, Japan) and fulvestrant was purchased from Tocris (Ellisville, MO). A $1 \mathrm{M}$ stock solution of each compound was prepared using $100 \%$ dimethyl sulfoxide (DMSO; Sigma-Aldrich, Ayrshire, UK) as a solvent and stored at $-20^{\circ} \mathrm{C}$ to avoid contamination. All antibodies, including ones specific for CaBP-9k (P-18), ER $\alpha$ (MC-20), progesterone receptor (PR) (C-19), glyceraldehyde-3-phosphate dehydroge- nase (GAPDH; A-3), goat anti-rabbit IgG (sc-362292), and goat anti-mouse IgG (sc-2005) were purchased from Santa Cruz Biotechnology, Inc. (Santa Cruz, CA).

Cell culture and treatment. GH3 cells were purchased from the Korean Cell Line Bank (Seoul, Korea) and maintained in Dulbecco's modified Eagle's medium (DMEM; Gibco-BRL, Grand Island, NY) with $10 \%$ fetal bovine serum (FBS; Gibco-BRL) plus penicillin/streptomycin (Gibco-BRL). The cells were incubated at $37^{\circ} \mathrm{C}$ in a humidified atmosphere containing $5 \% \mathrm{CO}_{2}$ and were passaged every 7 days using $0.25 \%$ trypsin with $1 \mathrm{mM}$ EDTA. In order to mitigate the effect of endogenous steroids, cells were cultured in phenol red-free medium containing 5\% (v/v) charcoal dextran-stripped serum (DMEM-5\% CD) for 7 days as previously described (30). The experimental treatments (DMSO as the negative control; $10^{-9}$ and $10^{-8} \mathrm{M}$ E2 as the positive control; $10^{-7}, 10^{-6}$ and $10^{-5} \mathrm{M}$ of BPA, NP, OP and IBP alone or in various combinations with equivalent concentrations of each compound) were performed in triplicate. The cells were harvested at a single end-point (24 h after treatment) to measure mRNA and protein levels.

To examine the mechanism of CaBP-9k induction by these EDCs, the cells were pre-treated with $10^{-7} \mathrm{M}$ fulvestrant for 30 min prior to EDC exposure as previously described (30). Following treatment with fulvestrant, the cells were exposed to a high dose $\left(10^{-5} \mathrm{M}\right)$ of BPA, NP, OP and IBP alone or combinations of these compounds $(\mathrm{BPA}+\mathrm{NP}, \mathrm{BPA}+\mathrm{NP}+\mathrm{OP}$ and $\mathrm{BPA}+\mathrm{NP}+\mathrm{IBP})$.

Reverse transcription-polymerase chain reaction (RT-PCR) analysis. For RT-PCR analysis, GH3 cells were grown in 6-well plates (Nunc, Roskilde, Denmark), and stimulated with the chemicals (alone or in combination) $24 \mathrm{~h}$ later. The cells were harvested by trypsinization and washed twice in Dulbecco's phosphate-buffered saline (DPBS; Gibco-BRL). Total RNA was isolated using TRI reagent (Ambion, Austin, TX) according to the manufacturer's instructions. Complementary DNA (cDNA) was then generated with M-MLV reverse transcriptase (Invitrogen, Carlsbad, CA) and 9-mer random primers (Takara Bio, Inc., Shiga, Japan). cDNA $(1 \mu \mathrm{l})$ was used for PCR at standard conditions: denaturation at $95^{\circ} \mathrm{C}$ for $30 \mathrm{sec}$, annealing at $55^{\circ} \mathrm{C}$ for $30 \mathrm{sec}$, and extension at $72^{\circ} \mathrm{C}$ for $1 \mathrm{~min}$. The following primers were used: cytochrome c oxidase subunit 1 (1A) forward, 5'-CCA GGG TTT GGA ATT ATT TC-3' and reverse, 5'-GAA GAT AAA CCC TAA GGC TC-3'; $C a B P-9 k$ forward, 5'-AAG AGC ATT TTT CAA AAA TA-3' and reverse, 5'-GTC TCA GAA TTT GCT TTA TT-3'; $P R$ forward, 5'-CAC AGG AGT TTG TCA AGG TC-3' and reverse, 5'-GGG ATT GGA TGA ACG TAT TC-3'; and $E R \alpha$ forward, 5'-GAC TTG AAT CTC CAC GAT CA-3' and reverse, 5'-CTT CAA GGT GCT GGA TAG AA-3'. The PCR products $(8 \mu \mathrm{l})$ were separated on a $2 \%$ agarose gel and stained with ethidium bromide. The gel was photographed, scanned and analyzed using the Quantity One program (Gel Doc EQ; Bio-Rad, Hercules, CA). The housekeeping $1 A$ gene was used for normalization. Data are shown as the average \pm SEM of 3 independent experiments.

Western blot analysis. Following treatment with the EDCs, the cells were rinsed twice with DPBS solution. Protein samples were extracted with Pro-prep solution (Intron Biotechnology, 
A
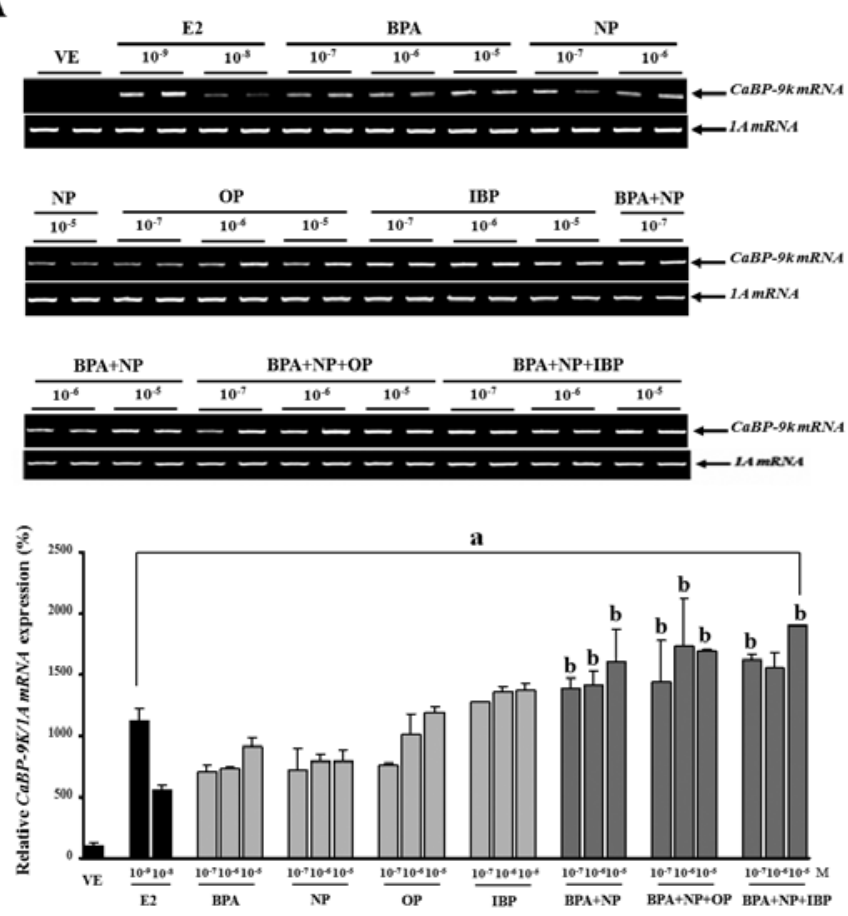

B
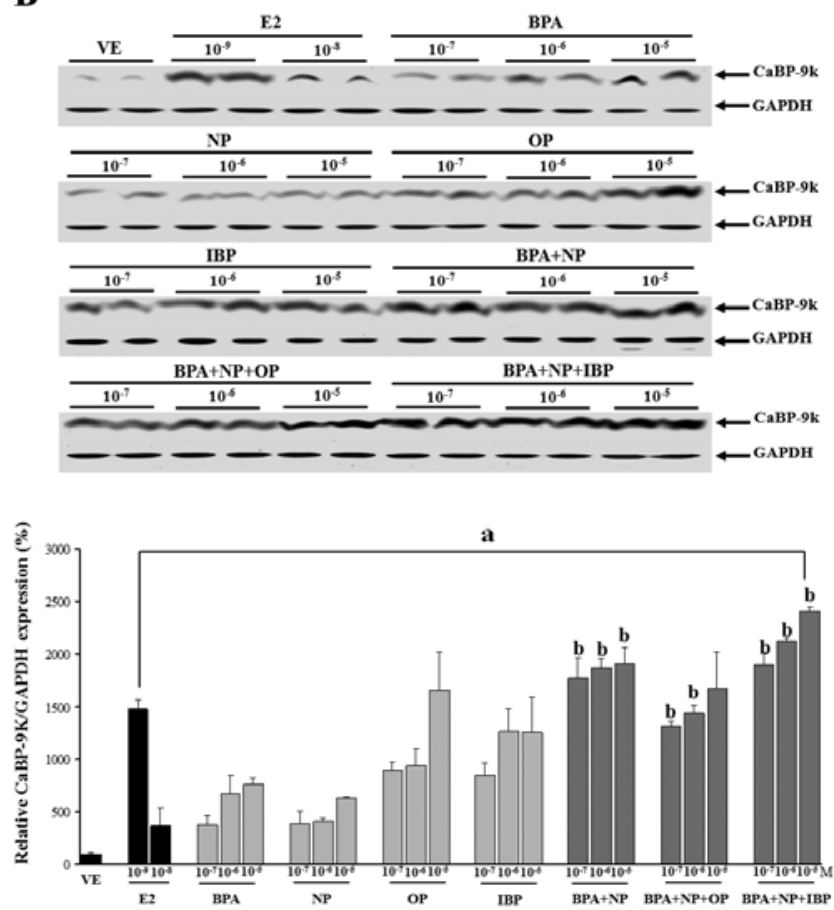

Figure 1. Effects of individual or combination treatment with BPA, NP, OP and IBP on CaBP-9k mRNA and protein expression. GH3 cells were treated with DMSO alone as the vehicle (VE); $10^{-9}$ and $10^{-8} \mathrm{M} \mathrm{E2}$ as the positive control. The cells were also treated with BPA, NP, OP and IBP alone (10 $0^{-7}, 10^{-6}$ and $\left.10^{-5} \mathrm{M}\right)$; a combination of BPA and NP $\left(10^{-7}, 10^{-6}\right.$ and $\left.10^{-5} \mathrm{M}\right)$; or combinations of BPA + NP + OP or BPA + NP + IBP $\left(10^{-7}, 10^{-6}\right.$ and $\left.10^{-5} \mathrm{M}\right)$. (A) $C a B P-9 k$ mRNA expression was measured by RT-PCR analysis. (B) CaBP-9k protein expression was evaluated by western blot analysis. Data represent the means \pm SEM of triplicate experiments. CaBP-9k expression was normalized to that of an internal control (1A gene for mRNA and GAPDH for protein). ${ }^{\text {a }}<0.05$ compared to the vehicle; ${ }^{b} \mathrm{P}<0.05$ comparing the combined treatment to treatment with each compound alone at the same concentration.

Seoul, Korea) following the manufacturer's instructions, and the total protein concentration of the supernatant was determined by a BCA protein assay (Pierce Chemical Co., Rockford, IL). Equal amounts of proteins were used for western blot analysis. Briefly, $30 \mu \mathrm{g}$ of cytosolic protein were subjected to electrophoresis on 7.5 and $12 \%$ SDS-PAGE gels. The separated proteins were transferred onto nitrocellulose membranes (Millipore, Bedford, MA). The membranes were washed twice with PBS containing $0.05 \%$ Tween-20 (PBS-T) and then incubated in blocking buffer (5\% non-fat milk; Difco, Franklin Lakes, NJ) for $2 \mathrm{~h}$ at room temperature. The membranes were then incubated with primary antibodies (anti-CaBP-9k, 1:500; anti-ER $\alpha$, 1:500; anti-PR, 1:500; and anti-GAPDH, 1:2,000) overnight at $4^{\circ} \mathrm{C}$. Incubation with secondary antibodies (diluted 1:3,000) was performed at room temperature for $1 \mathrm{~h}$. Antibody-bound proteins were visualized with the ECL chemiluminescent system (Amersham Pharmacia Biotech, Arlington, VA) and exposed to CP-BU NEW X-ray film (Agfa HealthCare NV, Mortsel, Belgium). Band intensities were measured using a molecular analysis program (Gel Doc 1000, version 1.5; Bio-Rad) and normalized to GAPDH levels as previously described (30).

Statistical analyses. Data were calculated as a percentage of the vehicle control (DMSO) and expressed as the means \pm standard error of the mean (SEM) of a single experiment performed in triplicate. Statistical significance was determined using one-way ANOVA followed by post hoc analysis (Tukey's range test). P-values $<0.05$ were considered to indicate statistically significant differences.

\section{Results}

Combined effects of BPA, NP, OP and IBP on CaBP-9k mRNA and protein expression in GH3 cells. EDCs can interfere with hormone signaling through various mechanisms. Some of these mechanisms are interrelated in a manner that may result in synergistic interactions. In this study, we examined the hypothesis that combined exposure to chemicals which function as hormone receptor agonists may result in greater additive toxicity. We performed experiments by assessing the effects of the chemicals (BPA, NP, OP and IBP) alone or in combination on CaBP-9k mRNA and protein expression in $\mathrm{GH} 3$ cells. Exposure of the cells to BPA, NP, OP and IBP alone or in combination, at concentrations which were equivalent to those of each chemical alone, significantly increased both CaBP-9k mRNA and protein expression in a dose-dependent manner. In particular, doses of the combined chemicals from $10^{-7}$ to $10^{-5} \mathrm{M}$ $(\mathrm{BPA}+\mathrm{NP}), 10^{-7}$ and $10^{-6} \mathrm{M}(\mathrm{BPA}+\mathrm{NP}+\mathrm{OP})$, or $10^{-7}$ and $10^{-5} \mathrm{M}(\mathrm{BPA}+\mathrm{NP}+\mathrm{IBP})$, significantly increased $\mathrm{CaBP}-9 \mathrm{k}$ gene expression compared to identical doses of each chemical alone.

These findings implied that these EDCs had a synergistic impact on estrogenic activity in the cells. The results from the experiments in which the cells were exposed to the single or combined treatment of BPA, NP, OP and IBP are presented in Fig. 1. 
A

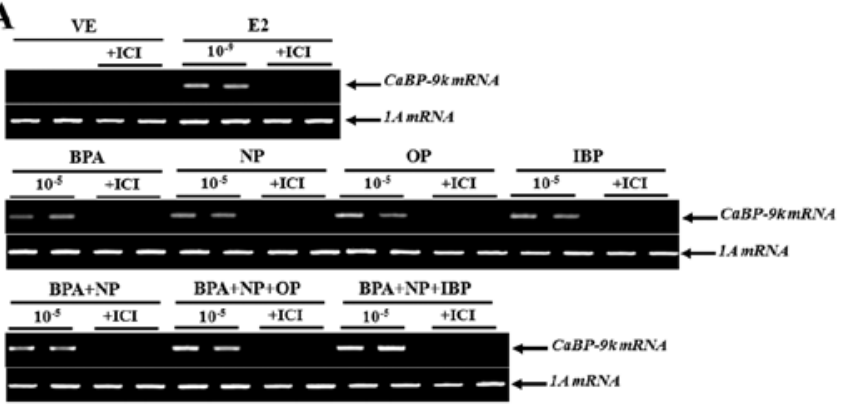

B

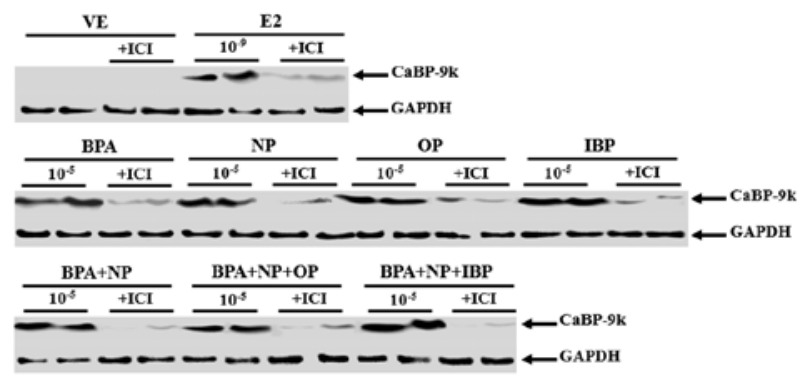

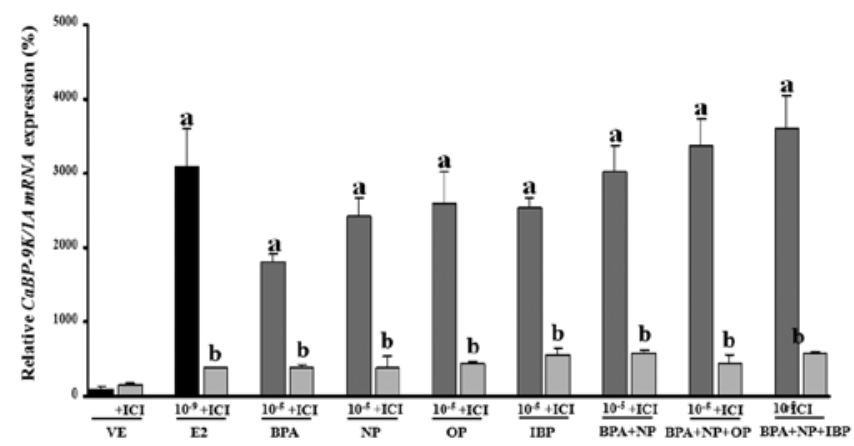

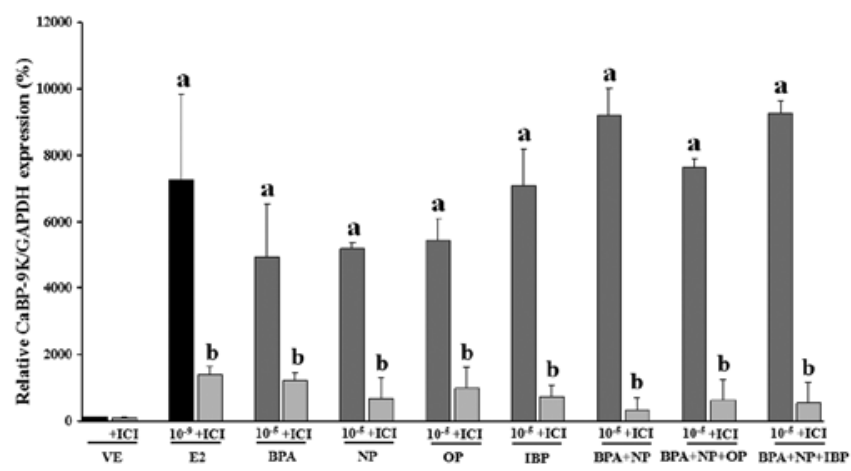

Figure 2. Effects of fulvestrant (ICI 182, 780) on the regulation of CaBP-9k mRNA and protein expression. GH3 cells were treated with DMSO alone as the vehicle (VE), $10^{-9} \mathrm{M} \mathrm{E2}$ as the positive control, or with $10^{-5} \mathrm{M}$ of BPA, NP, OP and IBP in combination or alone with or without a 30-min pre-treatment with fulvestrant $\left(10^{-7} \mathrm{M}\right)$. (A) mRNA expression of $\mathrm{CaBP}-9 \mathrm{k}$ was measured by RT-PCR analysis. (B) Protein expression of CaBP-9k was evaluated by western blot analysis. Data represent the means \pm SEM of triplicate experiments. CaBP-9k expression was normalized to that of an internal control (1A for mRNA and GAPDH for protein). ${ }^{a} \mathrm{P}<0.05$ compared to the vehicle; ${ }^{b} \mathrm{P}<0.05$ compared to single or combined chemical exposure.

Based on these findings, the cells were then treated with a high concentration $\left(10^{-5} \mathrm{M}\right)$ of the chemicals alone or in combination with or without fulvestrant (an anti-estrogen compound) to examine the mechanism underlying the synergistic effects of the examined chemicals (Fig. 2). The inhibition of CaBP-9k mRNA and protein expression following treatment with fulvestrant prior to treatment with the compounds (alone or in combination) was observed. These data suggest that the biological effects of BPA, NP, OP and IBP (alone or in combination) on $\mathrm{CaBP}-9 \mathrm{k}$ gene expression may involve an ER-mediated pathway in the GH3 cells.

Combined effects of BPA, NP, OP and IBP on PR mRNA and protein expression in $\mathrm{GH} 3$ cells. The effects of the single or combination treatment with EDCs (BPA, NP, OP and IBP) on PR mRNA and protein expression in the GH3 cells were evaluated. Four compounds elicited significant effects on both PR transcription and translation. Analysis of these results clearly showed that the EDC combinations at all doses, from $10^{-7}$ to $10^{-5} \mathrm{M}$ of $\mathrm{BPA}+\mathrm{NP}+\mathrm{OP}$ or BPA + NP + IBP increased PR mRNA expression compared to each chemical alone. High doses of BPA + NP $\left(10^{-6}\right.$ and $\left.10^{-5} \mathrm{M}\right), \mathrm{BPA}+\mathrm{NP}+\mathrm{OP}\left(10^{-5} \mathrm{M}\right)$ and $\mathrm{BPA}+\mathrm{NP}+\operatorname{IBP}\left(10^{-7}, 10^{-6}\right.$ and $\left.10^{-5} \mathrm{M}\right)$ also increased PR protein levels $(\mathrm{P}<0.05)$ compared to the exposure to each chemical alone (Fig. 3). The discrepancy observed between PR transcriptional and translational regulation may be explained by mRNA stability and/or protein accumulation. On the contrary, pre-treatment with fulvestrant completely blocked PR mRNA and protein expression (Fig. 4). These results were concomitant with those observed for CaBP-9k, suggesting that the expression of $C a B P-9 k$ and $P R$ genes was regulated in response to the combined effect of the estrogenic chemicals. In addition, the biological effects of the single or combined administration of BPA, NP, OP and IBP on CaBP-9k and PR expression may involve an ER-mediated pathway in GH3 cells.

Combined effects of BPA, NP, OP and IBP on ER mRNA and protein expression in $G H 3$ cells. In this study, no evidence of a synergistic effect on $E R \alpha$ mRNA and protein expression was observed when the EDCs were combined (Fig. 5). The expression levels of the ERa gene were upregulated when the cells were treated with fulvestrant prior to being treated with single or combined doses of EDCs (Fig. 6); however, these changes were not statistically significant (except for treatment with $10^{-5} \mathrm{M}$ IBP).

\section{Discussion}

There are a number of studies describing the potent estrogenic effects of BPA, NP, OP and IBP in vitro and in vivo $(24,30,31)$. The relative levels of the estrogenic activity of these chemicals have been reported as E2 $>\mathrm{NP}>\mathrm{OP}>\mathrm{BPA}$ (32). The ability of BPA to bind ERs is estimated to be 1/1,000 of E2 (33) and IBP showed an approximately 1,000-fold lower affinity than E2 (34). However, combinations of these EDCs at low concentrations used in consumer products have still shown estrogenic activities, and may be harmful to humans and animals (35). In a previous study, the combined effects of estrogens and xenoestrogens were shown to participate in non-genomic responses. The available experimental evidence showed that the effect 
A
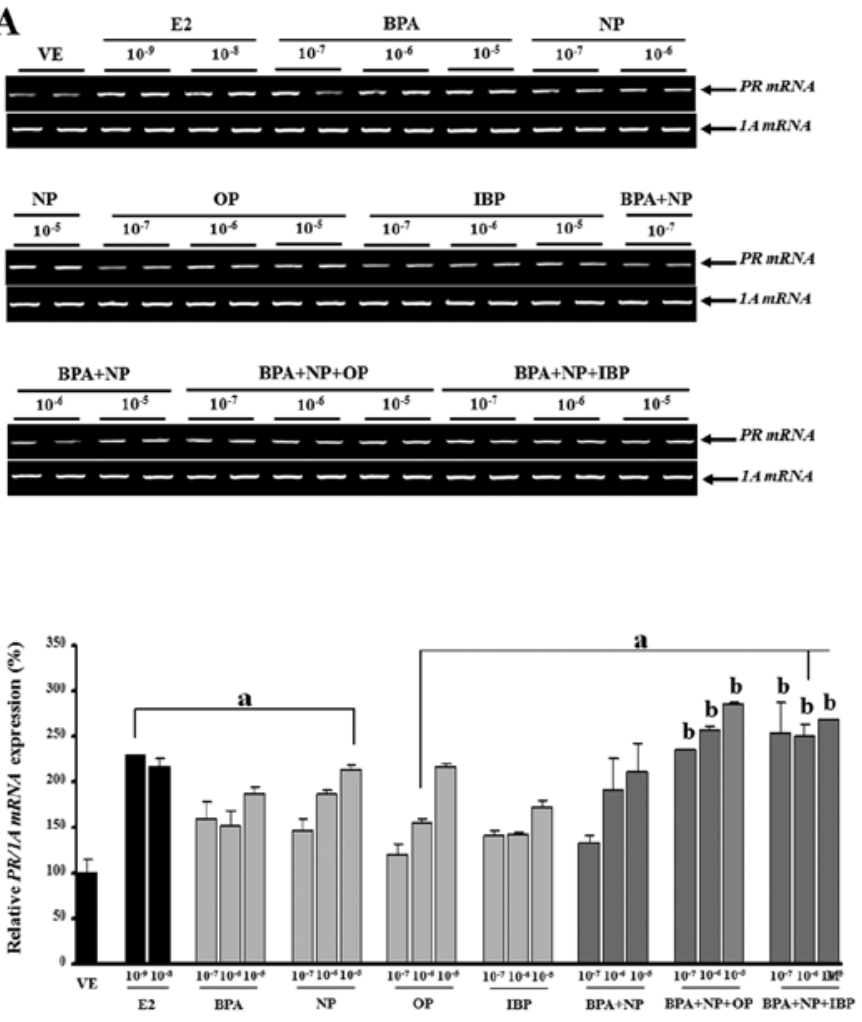

B

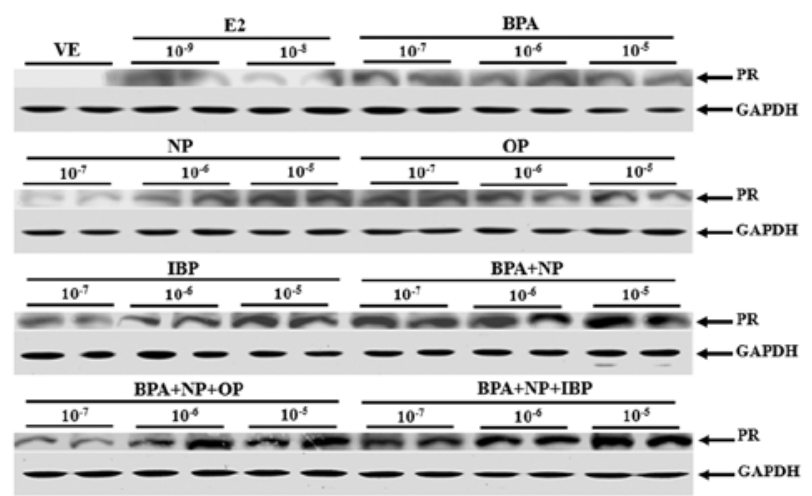

Figure 3. Effects of individual or combination treatment with BPA, NP, OP and IBP on PR mRNA and protein expression. GH3 cells were treated with DMSO alone as the vehicle (VE) or $10^{-9}$ and $10^{-8} \mathrm{M} \mathrm{E} 2$ as the positive control. The cells were also treated with BPA, NP, OP and IBP alone $\left(10^{-7}, 10^{-6}\right.$ and $\left.10^{-5} \mathrm{M}\right)$; a combination of BPA and NP $\left(10^{-7}, 10^{-6}\right.$ and $\left.10^{-5} \mathrm{M}\right)$; or combinations of BPA $+\mathrm{NP}+\mathrm{OP}$ or BPA + NP + IBP $\left(10^{-7}, 10^{-6}\right.$ and $\left.10^{-5} \mathrm{M}\right)$. (A) PR mRNA expression was evaluated by RT-PCR analysis. (B) PR protein expression was measured by western blot analysis. Data represent the means \pm SEM of triplicate experiments. PR expression was normalized to that of an internal control ( $1 A$ for mRNA and GAPDH for protein). ${ }^{\mathrm{a}} \mathrm{P}<0.05$ compared to the vehicle; ${ }^{\mathrm{b}} \mathrm{P}<0.05$ comparing combinations of the compounds to each compound alone at the same concentration.
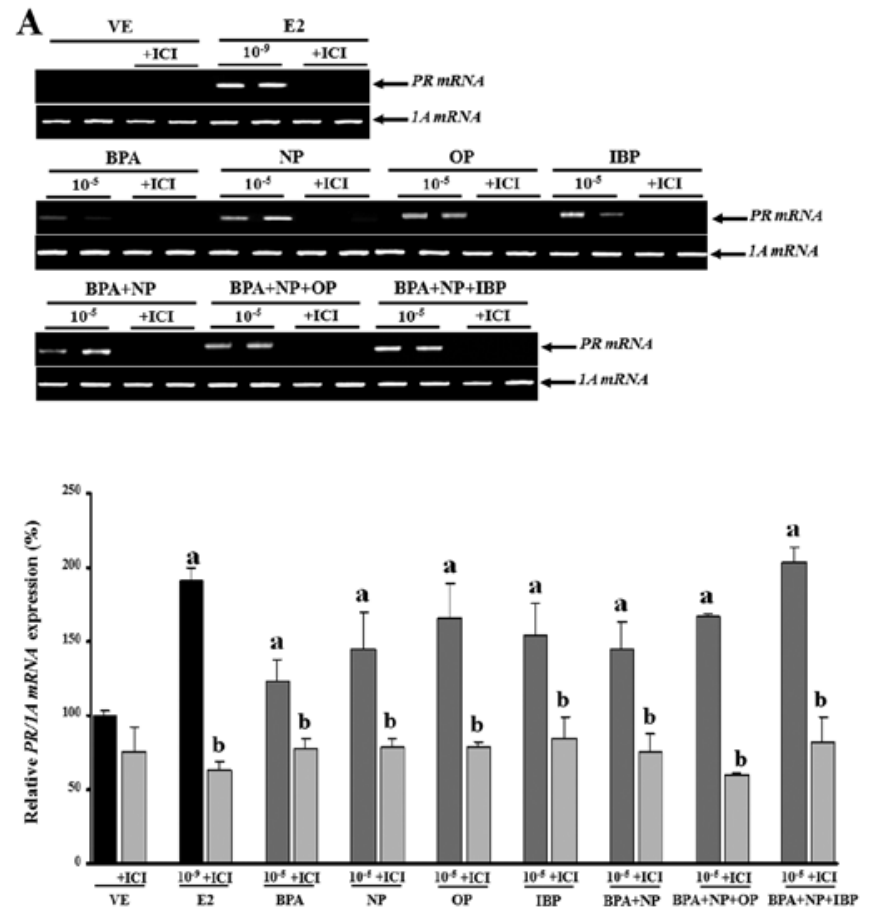

B
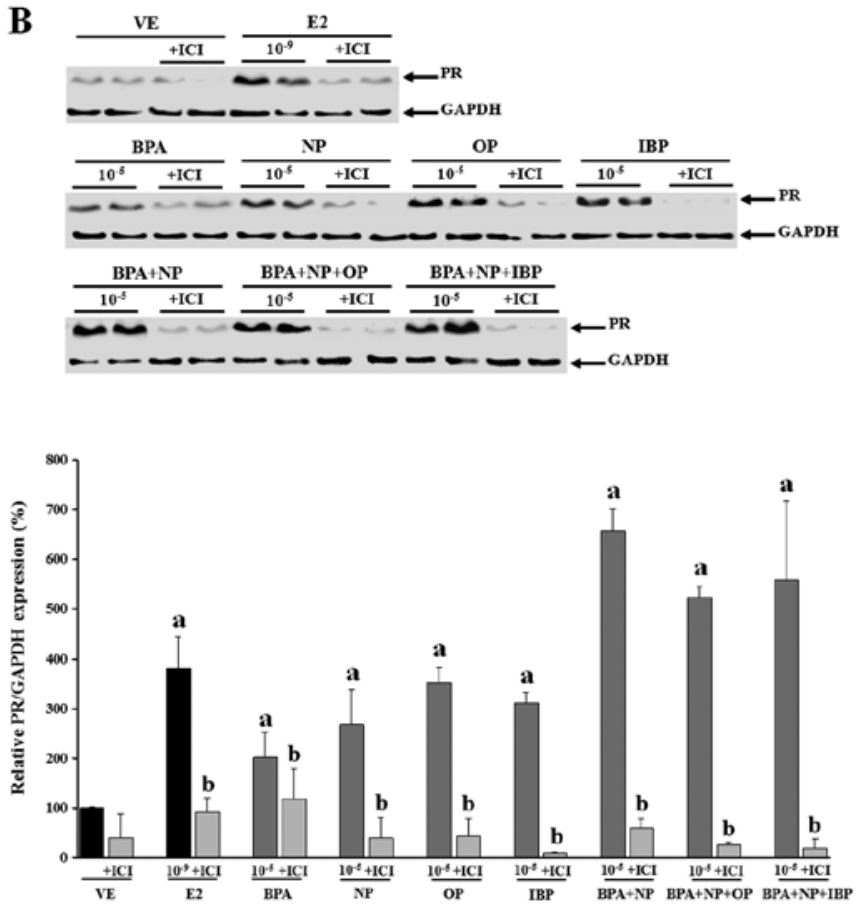

Figure 4. Effects of fulvestrant (ICI 182, 780) on the regulation of PR mRNA and protein expression. Cells were treated with DMSO alone as the vehicle (VE), with $10^{-9} \mathrm{ME} 2$ as the positive control, or with $10^{-5} \mathrm{M}$ of BPA, NP, OP and IBP alone or in combination with or without a 30-min pre-treatment with fulvestrant $\left(10^{-7} \mathrm{M}\right)$. (A) PR mRNA expression was measured by RT-PCR analysis. (B) PR protein expression was evaluated by western blot analysis. Data represent the means \pm SEM of triplicate experiments. PR expression was normalized to that of an internal control ( $1 \mathrm{~A}$ for mRNA and GAPDH for protein). ${ }^{\mathrm{a}} \mathrm{P}<0.05$ compared to the vehicle; ${ }^{b} \mathrm{P}<0.05$ compared to single or combined chemical exposure. 
A
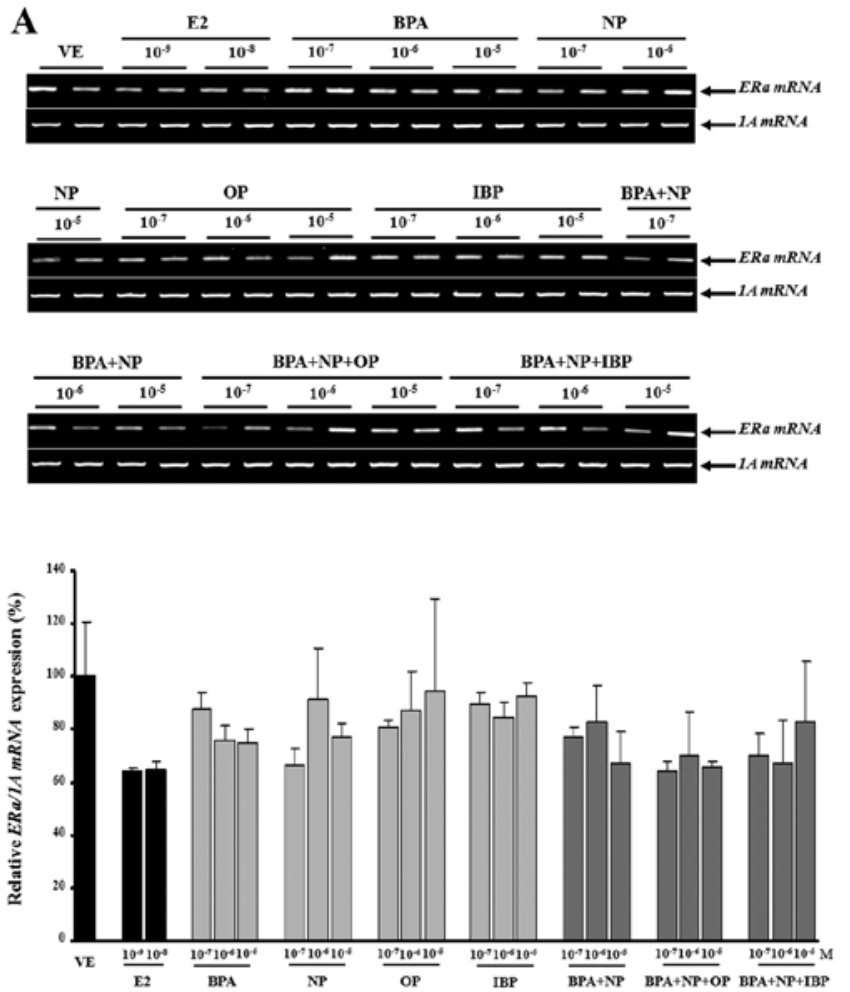
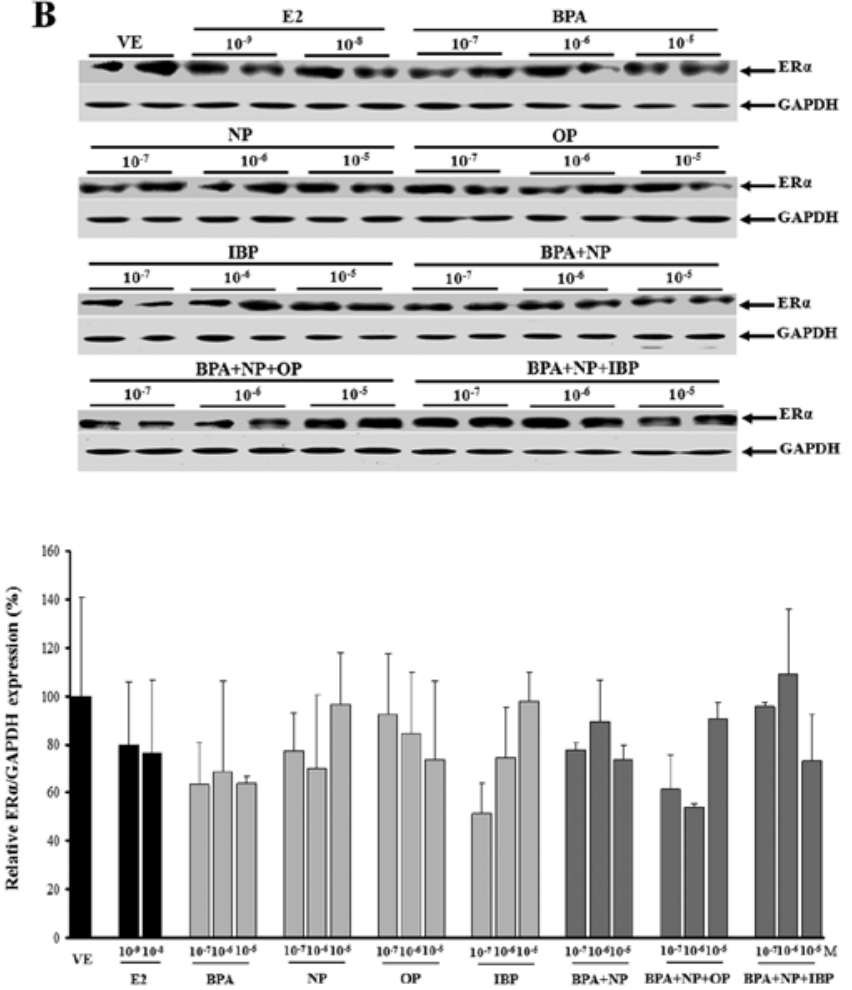

Figure 5. Effects of individual or combination treatment with BPA, NP, OP and IBP on ER $\alpha$ mRNA and protein expression. GH3 cells were treated with DMSO alone as the vehicle (VE), or with $10^{-9}$ and $10^{-8} \mathrm{M}$ E2 as the positive control. The cells were also treated with BPA, NP, OP and IBP alone (10 ${ }^{-7}, 10^{-6}$ and $\left.10^{-5} \mathrm{M}\right)$; a combination of BPA and NP $\left(10^{-7}, 10^{-6}\right.$ and $\left.10^{-5} \mathrm{M}\right)$; or combinations of BPA + NP + OP or BPA + NP + IBP $\left(10^{-7}, 10^{-6}\right.$ and $\left.10^{-5} \mathrm{M}\right)$. (A) ERa mRNA expression was measured by RT-PCR analysis. (B) ER $\alpha$ protein expression was evaluated by western blot analysis. Data represent the means \pm SEM of triplicate experiments. ER $\alpha$ expression was normalized to that of an internal control (1A for mRNA and GAPDH for protein).
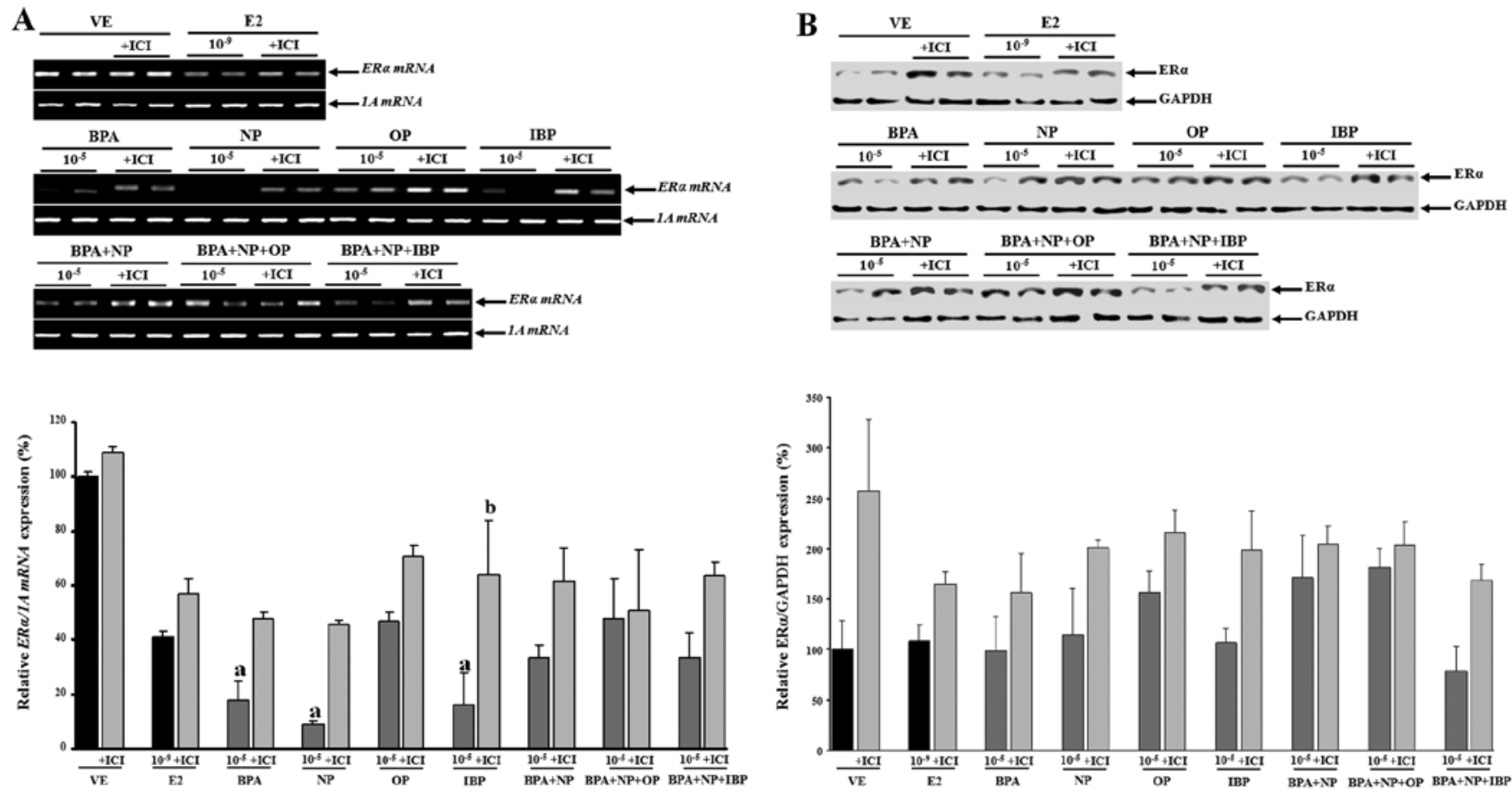

Figure 6. Effects of fulvestrant (ICI 182,780) on the regulation of ER $\alpha$ mRNA and protein expression. GH3 cells were treated with DMSO alone as the vehicle (VE), $10^{-9} \mathrm{M} \mathrm{E} 2$ as the positive control, or $10^{-5} \mathrm{M}$ of BPA, NP, OP and IBP alone or in combination with or without a 30-min pre-treatment with fulvestrant $\left(10^{-7} \mathrm{M}\right)$. (A) $E R \alpha$ mRNA expression was evaluated by RT-PCR analysis. (B) ER protein expression was measured by western blot analysis. Data represent the means \pm SEM of triplicate experiments. ER $\alpha$ expression was normalized to that of an internal control ( $1 A$ for mRNA and GAPDH for protein). ${ }^{a}<0.05$ compared to the vehicle; ${ }^{\mathrm{b}} \mathrm{P}<0.05$ compared to single or combined chemical exposure. 
of low-dose combinations may result in biological perturbation (36). Rajapakse et al (2) examined a combination of 11 xenoestrogens at levels close to their individual no observed effect concentrations (NOECs) and suggested doubling the dose of E2. Combinations of weak xenoestrogens (hydroxylated PCBs, benzophenones, parabenes, BPA and genistein) at low levels that individually have undetectable effects are able to create an impact on the actions of endogenous estrogens in body fluids and tissues (35). Furthermore, the combined estrogenic effects of EDCs are a risk factor for breast cancer in women (37).

Humans are exposed to a variety and combinations of natural and synthetic compounds that may influence various endocrine processes. From a hazard and risk assessment point of view, it is important to know whether exposure to combinations of these compounds results in additive, antagonistic, or synergistic actions (38). Very few studies have addressed the estrogenic effects of simultaneous exposure to 2 or 3 different EDCs. Our previous studies indicated that combined exposure to OP and IBP (20) or multiple parabens (21) at low concentrations synergistically affects the induction of $\mathrm{CaBP}-9 \mathrm{k}$ gene expression via an ER- or PR-dependent pathway. In the present study, we expanded the scope of our research to examine the synergistic effects of BPA, NP, OP and IBP on estrogenic activity in a rat pituitary cell line. The estrogenic effects of BPA + NP, BPA $+\mathrm{NP}+\mathrm{OP}$ and $\mathrm{BPA}+\mathrm{NP}+\mathrm{IBP}$ combinations on $\mathrm{CaBP}-9 \mathrm{k}$ mRNA and protein expression were dose-dependent and more potent than those of the individual compounds administered at the equivalent concentrations. An endogenous gene expression assay that measures estrogen-induced alterations has been created and has been used extensively to detect the estrogenic activity of EDCs in vitro and in vivo (39). In previous studies, CaBP-9k was found to be expressed in a variety of mammalian tissues including the uterus, placenta, intestine, kidney, pituitary gland and bone $(23,25,28,40)$. It has also been shown that CaBP-9k gene expression is highly modulated by E2 in rats, mice and dogs (24-26,30,31), suggesting that this gene may be used as a biomarker for evaluating the estrogenicity of putative estrogenic compounds (23). In the present study, our results showed that there was a synergistic effect when 2 or 3 estrogenic chemicals (BPA, NP, OP and IBP) were administered in combination. Based on our findings, we also suggest that the $C a B P-9 k$ gene is a suitable biomarker to assess the variation in the estrogenic activity of combined EDCs.

A previous study has demonstrated that a broad range of EDCs is capable of interacting with ERs and inducing ER-mediated responses (41). Cellular levels of estrogens regulate development and growth by inducing differentiation and cell proliferation classically through ERs by stimulating the transcription of target genes. The disruption of ER signaling pathways can contribute to adverse health effects, such as developmental reproduction abnormalities and endocrinerelated cancers (42). To better understand the molecular events promoted by the administration of a combination of EDCs in this study, fulvestrant was used to examine the potential involvement of ER signaling in the individual or combined action of the compounds. The levels of CaBP-9k mRNA and protein were significantly increased in a synergistic manner, whereas pre-treatment with fulvestrant completely attenuated the single or combined effects on $C a B P-9 k$ gene expression.
Of note, we found that the patterns of $P R$ gene expression were similar to those of $C a B P-9 k$ in response to individual and combined chemical exposure. Pre-treatment with fulvestrant completely blocked $P R$ expression. These results suggest that the single and combined effects of BPA, NP, OP and IBP require an ER-mediated signaling pathway. Although there were fluctuations in ER $\alpha$ mRNA and protein expression levels after treatment with these EDCs, no synergistic effect compared to the vehicle or each individual chemical was observed. In addition, the levels of $E R \alpha$ gene expression were not altered when the cells were pre-treated with fulvestrant.

In a previous study, treatment with industrial compounds (BPA, NP and OP) increased the expression of $P R$ mRNA in the frontal cortex of adult ovariectomized rats (43). In CD-1 mouse uterus, exposure to 25 and $250 \mathrm{ng} \mathrm{BPA} / \mathrm{kg}$ body weight/ day resulted in increased PR expression in the endometrium and subepithelial stroma (44). Okubo et al (45) found that MCF-7 cells treated with IBP exhibited a decrease in ER $\alpha$ gene expression while PR expression was enhanced. In addition, observing the involvement of ERs in paraben-induced responses has increased the understanding of the mechanism(s) underlying molecular events promoted by estrogenic compounds (25). Other studies have indicated that CaBP-9k expression is responsive to E2 via ER signaling in the rat uterus, ovary, and $\mathrm{GH} 3$ cells in which $\mathrm{ER} \alpha, \mathrm{ER} \beta$ and PR are expressed $(21,25,46)$.

In conclusion, the results of the present study provide evidence that combinations of BPA, NP, OP and IBP have synergistic effects on estrogenic activity in GH3 cells. In addition, this synergistic activity of the EDCs is induced via an ER-mediated signaling pathway. We also suggest that the $C a B P-9 k$ gene is an appropriate biomarker for evaluating the synergistic actions of EDCs in vitro. However, studies with wider concentration ranges and multiple combinations of EDCs in vivo and in vitro are warranted to determine the actual profile of xenoestrogenic activities.

\section{Acknowledgements}

This study was supported by a grant (12182KFDA638) from the Korea Food and Drug Administration. T.T.B.V. received funding from the Vietnam National Foundation for Science and Technology Development (NAFOSTED) no. 106.06-2011.33.

\section{References}

1. Sehulster L and Chinn RY; CDC; HICPAC: Guidelines for environmental infection control in health-care facilities. Recommendations of CDC and the Healthcare Infection Control Practices Advisory Committee (HICPAC). MMWR Recomm Rep 52: 1-42, 2003.

2. Rajapakse N, Silva E and Kortenkamp A: Combining xenoestrogens at levels below individual no-observed-effect concentrations dramatically enhances steroid hormone action. Environ Health Perspect 110: 917-921, 2002.

3. Jin S, Yang F, Liao T, Hui Y, Wen S and Xu Y: Enhanced effects by mixtures of three estrogenic compounds at environmentally relevant levels on development of Chinese rare minnow (Gobiocypris rarus). Environ Toxicol Pharmacol 33: 277-283, 2011.

4. Brian JV, Harris CA, Scholze M, et al: Accurate prediction of the response of freshwater fish to a mixture of estrogenic chemicals. Environ Health Perspect 113: 721-728, 2005.

5. Poongothai S, Ravikrishnan R and Murthy PB: Endocrine disruption and perspective human health implications: a review. Internet J Toxicol 4: 2, 2008, doi: 10.5580/263. 
6. Olea N, Arrebola JP, Taoufiki J, Fernández-Valades R, Prada R, Navea N, Molina-Molina JM and Fernandez MF: Alkylphenols and bisphenol-A and its chlorinated derivatives in adipose tissue of children. Environ Toxicol 110: 369, 2008.

7. Soni MG, Carabin IG and Burdock GA: Safety assessment of esters of p-hydroxybenzoic acid (parabens). Food Chem Toxicol 43: 985-1015, 2005.

8. Roy D, Colerangle JB and Singh KP: Is exposure to environmental or industrial endocrine disrupting estrogen-like chemicals able to cause genomic instability? Front Biosci 3: d913-d921, 1998.

9. Tayama S, Nakagawa Y and Tayama K: Genotoxic effects of environmental estrogen-like compounds in CHO-K1 cells. Mutat Res 649: 114-125, 2008

10. Rhee JS, Lee YM, Raisuddin S and Lee JS: Expression of R-ras oncogenes in the hermaphroditic fish Kryptolebias marmoratus, exposed to endocrine disrupting chemicals. Comp Biochem Physiol C Toxicol Pharmacol 149: 433-439, 2009.

11. Lee YM, Raisuddin S, Rhee JS, Ki JS, Kim IC and Lee JS: Modulatory effect of environmental endocrine disruptors on N-ras oncogene expression in the hermaphroditic fish, Kryptolebias marmoratus. Comp Biochem Physiol C Toxicol Pharmacol 147: 299-305, 2008.

12. Baba K, Okada K, Kinoshita T and Imaoka S: Bisphenol A disrupts Notch signaling by inhibiting gamma-secretase activity and causes eye dysplasia of Xenopus laevis. Toxicol Sci 108 . 344-355, 2009.

13. Kudo C, Wada K, Masuda T, et al: Nonylphenol induces the death of neural stem cells due to activation of the caspase cascade and regulation of the cell cycle. J Neurochem 88: 1416-1423, 2004.

14. Sato K, Matsuki N, Ohno Y and Nakazawa K: Effects of 17beta-estradiol and xenoestrogens on the neuronal survival in an organotypic hippocampal culture. Neuroendocrinology 76 : 223-234, 2002

15. Blair RM, Fang H, Branham WS, et al: The estrogen receptor relative binding affinities of 188 natural and xenochemicals: structural diversity of ligands. Toxicol Sci 54: 138-153, 2000.

16. Harvey PW: Parabens, oestrogenicity, underarm cosmetics and breast cancer: a perspective on a hypothesis. J Appl Toxicol 23: 285-288, 2003.

17. Darbre PD, Byford JR, Shaw LE, Horton RA, Pope GS and Sauer MJ: Oestrogenic activity of isobutylparaben in vitro and in vivo. J Appl Toxicol 22: 219-226, 2002.

18. Lv X,Zhou Q, Song M, Jiang G and Shao J: Vitellogenic responses of 17beta-estradiol and bisphenol A in male Chinese loach (Misgurnus anguillicaudatus). Environ Toxicol Pharmacol 24 $155-159,2007$.

19. Kaneko M, Okada R, Yamamoto K, et al: Bisphenol A acts differently from and independently of thyroid hormone in suppressing thyrotropin release from the bullfrog pituitary. Gen Comp Endocrinol 155: 574-580, 2008.

20. Kim YR, Jung EM, Choi KC and Jeung EB: Synergistic effects of octylphenol and isobutyl paraben on the expression of calbindin-D ${ }_{9 \mathrm{k}}$ in GH3 rat pituitary cells. Int J Mol Med 29: 294-302, 2012.

21. Yang H, Nguyen TT, An BS, Choi KC and Jeung EB: Synergistic effects of parabens on the induction of calbindin-D9k gene expression act via a progesterone receptor-mediated pathway in GH3 cells. Hum Exp Toxicol 31: 134-144, 2012.

22. Shen HY, Jiang HL, Mao HL, Pan G, Zhou L and Cao YF: Simultaneous determination of seven phthalates and four parabens in cosmetic products using HPLC-DAD and GC-MS methods. J Sep Sci 30: 48-54, 2007.

23. Choi KC and Jeung EB: Molecular mechanism of regulation of the calcium-binding protein calbindin- $\mathrm{D}_{9 \mathrm{k}}$, and its physiological role(s) in mammals: a review of current research. J Cell Mol Med 12: 409-420, 2008.

24. An BS, Kang SK, Shin JH and Jeung EB: Stimulation of calbindin-D(9k) mRNA expression in the rat uterus by octyl-phenol, nonylphenol and bisphenol. Mol Cell Endocrinol 191: 177-186, 2002.

25. Vo TT and Jeung EB: An evaluation of estrogenic activity of parabens using uterine calbindin-d9k gene in an immature rat model. Toxicol Sci 112: 68-77, 2009

26. Ji YK, Lee GS, Choi KC and Jeung EB: Anti-progestogenic effect of flutamide on uterine expression of calbindin-D9k mRNA and protein in immature mice. Reprod Toxicol 22: 694-701, 2006.

27. Shin JH, Moon HJ, Kang IH, et al: Calbindin-D9k mRNA expression in the rat uterus following exposure to methoxychlor: a comparison of oral and subcutaneous exposure. J Reprod Dev 53: $179-188,2007$
28. Lee GS, Choi KC and Jeung EB: Glucocorticoids differentially regulate expression of duodenal and renal calbindin-D9k through glucocorticoid receptor-mediated pathway in mouse model. Am J Physiol Endocrinol Metab 290: E299-E307, 2006.

29. Lee GS, Choi KC, Kim HJ and Jeung EB: Effect of genistein as a selective estrogen receptor beta agonist on the expression of Calbindin-D9k in the uterus of immature rats. Toxicol Sci 82: 451-457, 2004.

30. Vo TT, Jung EM, Choi KC, Yu FH and Jeung EB: Estrogen receptor alpha is involved in the induction of Calbindin-D $(9 \mathrm{k})$ and progesterone receptor by parabens in $\mathrm{GH} 3$ cells: a biomarker gene for screening xenoestrogens. Steroids 76: 675-681, 2011.

31. Dang VH, Nguyen TH, Choi KC and Jeung EB: A calciumbinding protein, calbindin-D9k, is regulated through an estrogen-receptor mediated mechanism following xenoestrogen exposure in the GH3 cell line. Toxicol Sci 98: 408-415, 2007.

32. Song M, Xu Y, Jiang Q, et al: Measurement of estrogenic activity in sediments from Haihe and Dagu River, China. Environ Int 32: 676-681, 2006

33. Takayanagi S, Tokunaga T, Liu X, Okada H, Matsushima A and Shimohigashi Y: Endocrine disruptor bisphenol A strongly binds to human estrogen-related receptor gamma (ERRgamma) with high constitutive activity. Toxicol Lett 167: 95-105, 2006.

34. Vo TT, Yoo YM, Choi KC and Jeung EB: Potential estrogenic effect(s) of parabens at the prepubertal stage of a postnatal female rat model. Reprod Toxicol 29: 306-316, 2010.

35. Silva E, Rajapakse N and Kortenkamp A: Something from 'nothing' - eight weak estrogenic chemicals combined at concentrations below NOECs produce significant mixture effects Environ Sci Technol 36: 1751-1756, 2002.

36. Kortenkamp A: Low dose mixture effects of endocrine disrupters: implications for risk assessment and epidemiology. Int J Androl 31: 233-240, 2008.

37. Ibarluzea JM, Fernández MF, Santa-Marina L, Olea-Serrano MF, Rivas AM, Aurrekoetxea JJ, Expósito J, Lorenzo M, Torné P, Villalobos M, Pedraza V, Sasco AJ and Olea N: Breast cancer risk and the combined effect of environmental estrogens. Cancer Causes Control 15: 591-600, 2004.

38. Stewart AG and Carter J: Towards the development of a multidisciplinary understanding of the effects of toxic chemical mixtures on health. Environ Geochem Health 31: 239-251, 2009.

39. An BS, Choi KC, Kang SK, Hwang WS and Jeung EB: Novel Calbindin-D(9k) protein as a useful biomarker for environmental estrogenic compounds in the uterus of immature rats. Reprod Toxicol 17: 311-319, 2003

40. Tinnanooru P, Dang VH, Nguyen TH, Lee GS, Choi KC and Jeung EB: Estrogen regulates the localization and expression of calbindin-D9k in the pituitary gland of immature male rats via the ERalpha-pathway. Mol Cell Endocrinol 285: 26-33, 2008.

41. Bonefeld-Jorgensen EC, Long M, Hofmeister MV and Vinggaard AM: Endocrine-disrupting potential of bisphenol A, bisphenol A dimethacrylate, 4-n-nonylphenol, and 4-n-octylphenol in vitro: new data and a brief review. Environ Health Perspect 115 (Suppl 1): S69-S76, 2007.

42. Ghisari M and Bonefeld-Jorgensen EC: Effects of plasticizers and their mixtures on estrogen receptor and thyroid hormone functions. Toxicol Lett 189: 67-77, 2009.

43. Funabashi T, Nakamura TJ and Kimura F: p-Nonylphenol, 4-tert-octylphenol and bisphenol A increase the expression of progesterone receptor mRNA in the frontal cortex of adult ovariectomized rats. J Neuroendocrinol 16: 99-104, 2004.

44. Markey CM, Wadia PR, Rubin BS, Sonnenschein C and Soto AM: Long-term effects of fetal exposure to low doses of the xenoestrogen bisphenol-A in the female mouse genital tract. Biol Reprod 72: 1344-1351, 2005.

45. Okubo T, Yokoyama Y, Kano K and Kano I: ER-dependent estrogenic activity of parabens assessed by proliferation of human breast cancer MCF-7 cells and expression of ERalpha and PR. Food Chem Toxicol 39: 1225-1232, 2001.

46. Lee GS, Kim HJ, Jung YW, Choi KC and Jeung EB: Estrogen receptor alpha pathway is involved in the regulation of Calbindin-D9k in the uterus of immature rats. Toxicol Sci 84: 270-277, 2005 